\title{
Massive Migration von Silikonöl in den subkonjunktivalen Raum: Ein Dilemma von Leckage-Mechanismen
}

\author{
Jesús Téllez ${ }^{\mathrm{a}, \mathrm{c}}$ José I. Vela ${ }^{\mathrm{a}, \mathrm{b}}$ Sabina Luna ${ }^{\mathrm{a}, \mathrm{c}}$ Rubén Delgado ${ }^{\mathrm{a}}$ \\ aDepartment of Ophthalmology, Hospital de la Santa Creu I de Sant Pau, Autonoma University of Barcelona, Barcelona, Spanien; \\ ${ }^{b}$ Departmen of Ophthalmology, Institut Condal d'Oftamologia, Barcelona, Spanien; ' Department of Ophthalmology, \\ Grupo Admiravisión, Barcelona, Spanien
}

\author{
Schlüsselwörter \\ Glaukomdrainagesystem · Migration von Silikonöl \\ subkonjunktivaler Raum · nahtlose Vitrektomie
}

\begin{abstract}
Zusammenfassung
Zielsetzung: Wir berichten über einen Fall von früh aufgetretener massiver Migration von Silikonöl in den subkonjunktivalen Raum nach nahtloser Vitrektomie bei einem Patienten mit vorheriger Pars-plana-Implantation eines Glaukomdrainagesystems.

Fallbericht: Ein 80-jähriger Mann stellte sich mit einem neovaskulären Glaukom aufgrund einer proliferativen diabetischen Retinopathie im linken Auge vor. Nach intrakameraler Injektion von Bevacizumab und panretinaler Photokoagulation wurde bei dem Patienten eine 23-Gauge Pars-plana-Vitrektomie (PPV) in Kombination mit einer superotemporalen Pars-plana-Implantation einer Ahmed Glaucoma Valve durchgeführt. Im weiteren Verlauf erfolgte eine
\end{abstract}

erneute 23-Gauge-PPV wegen einer dichten Glaskörperblutung. Um wiederkehrende intraokuläre Blutungen zu verhindern, wurde intravitreal Silikonöl mit einer Viskosität von 1000 Zentistokes eingebracht. Es erfolgte kein Nahtverschluss. Im ersten postoperativen Monat kam es zu einer massiven Migration von Silikonöl in den $360^{\circ}$ umfassenden subkonjunktivalen Raum, bis kein intraokuläres Silikonöl mehr nachweisbar war.

Schlussfolgerungen: Wir erörtern die in diesem speziellen Fall möglichen Leckage-Mechanismen.

(c) 2018 The Author(s), Published by S. Karger AG, Basel

\section{Einführung}

Das neovaskuläre Glaukom (NVG) ist das Endstadium ischämischer retinaler Gefäßerkrankungen wie der proliferativen diabetischen Retinopathie oder dem retinalen Zentralvenenverschluss. Silikonöl wird bei diesen Patienten als Zusatztherapie für die interne Tamponade im Rahmen von Glaskörper- und Retina-Operationen verwendet. Zudem erfordert das therapierefraktäre NVG häufig die Implantation eines Glaukomdrainagesystems (glaucoma drainage device, GDD). Dabei erfolgt nicht selten eine Pars-plana-Vitrektomie (PPV) in Kombination mit einer Silikonöl-Injektion und Implantation eines Glaukomdrainagesystems. In der vorliegenden Arbeit berichten wir über einen Fall von früh aufgetretener massiver Migration von Silikonöl in den subkonjunktivalen Raum nach nahtloser PPV bei einem Patienten mit vorheriger Implantation eines Pars-plana-GDD, und wir erörtern die möglichen Leckage-Mechanismen.

\section{KARGER \\ Fax +4976145207 14 \\ information@karger.com}

www.karger.com
(๑) 2019 S. Karger GmbH, Freiburg

Accessible online at: www.karger.com/kop
José I. Vela

C/ Aragó $4694^{\circ} 5^{\circ}$

08013 Barcelona, Spanien

34218jvs@gmail.com 


\section{Fallbericht}

Ein 80-jähriger Mann ohne bekannte Vorerkrankungen stellte sich mit einem NVG aufgrund einer proliferativen diabetischen Retinopathie im linken pseudophaken Auge vor. Nach intrakameraler Injektion von Bevacizumab und panretinaler Photokoagulation wurde bei dem Patienten eine 23-Gauge-PPV in Kombination mit einer superotemporalen Pars-plana-Implantation einer Ahmed Glaucoma Valve durchgeführt. Die Operation erfolgte mittels «Sklera-Patch-Technik», d.h. dass ein fornixbasierter Konjunktivalappen geschaffen und das Röhrchen in die Glaskörperhöhle eingelegt wurde. Dadurch kam es zu einer vollständigen Kontrolle des intraokularen Drucks (intraocular pressure, IOP). Fünfzehn Monate nach der Operation wurde eine erneute 23-Gauge-PPV wegen einer dichten Glaskörperblutung durchgeführt. Zur Verhinderung wiederkehrender intraokulärer Blutungen wurde intravitreal Silikonöl mit einer Viskosität von 1000 Zentistokes eingebracht. Es erfolgte kein Nahtverschluss. Im ersten postoperativen Monat kam es zu einer progredienten Migration von Silikonöl in den $360^{\circ}$ umfassenden subkonjunktivalen Raum, bis kein intraokuläres Silikonöl mehr nachweisbar war (Abb. 1). Der Patient zeigte keine Symptome und die bestkorrigierte Sehschärfe (best corrected visual acuity, BCVA) lag weiterhin bei «keine Lichtwahrnehmung» im linken Auge. Zum Ausschluss von Silikonöl in der Orbita erfolgte eine Magnetresonanztomographie. Einen Monat nach der Operation wurde der Versuch unternommen, das gesamte Silikonöl aus dem subkonjunktivalen Raum zu entfernen, was jedoch nur unvollständig gelang (Abb. 2). Die intraoperative Überprüfung der Sklerotomien ergab keinen Hinweis auf eine Leckage.

\section{Diskussion}

Die intraokulare Silikonöl-Migration in den subkonjunktivalen Raum durch ein Glaukom-Drainageröhrchen (Shunt) oder durch nahtlose Sklerotomien wurde bereits in früheren Arbeiten beschrieben [1-7]. Da im vorliegenden Fall sowohl eine nahtlose PPV als auch eine Pars-plana-Implantation eines Glaukomdrainagesystems durchgeführt wurde, stellt sich die Frage: Wie ist das Silikonöl in den subkonjunktivalen Raum gelangt?

Interessanterweise trat die massive Silikonöl-Extravasation bereits innerhalb eines Monats nach Einbringung auf, ohne dass es zu einer Emulsifikation des Öls gekommen war. Ferner ist es ungewöhnlich, dass das Silikonöl von Beginn der Migration an die gesamte Zirkumferenz von $360^{\circ}$ des subkonjunktivalen Raums umfasste und nicht auf das Sickerkissen oder den Bereich um die Ahmed-Valve begrenzt blieb. Die langsame Migration von emulsifiziertem Silikonöl durch eine reife GDD-Kapsel und die Infiltration von konjunktivalem Gewebe durch Silikonöl wurde in der Vergangenheit für nicht-restriktive Implantate beschrieben $[1,2]$. Außerdem wurde ein seltener Fall von frühzeitiger massiver subkonjunktivaler und orbitaler Migration durch eine Ahmed Glaucoma Valve berichtet, die auftritt, wenn das Silikonöl in Kontakt mit dem Röhrchen kommt und die Kapsel noch nicht vollständig ausgebildet ist [3]. Bei unserem Patienten hatte sich die Kapsel

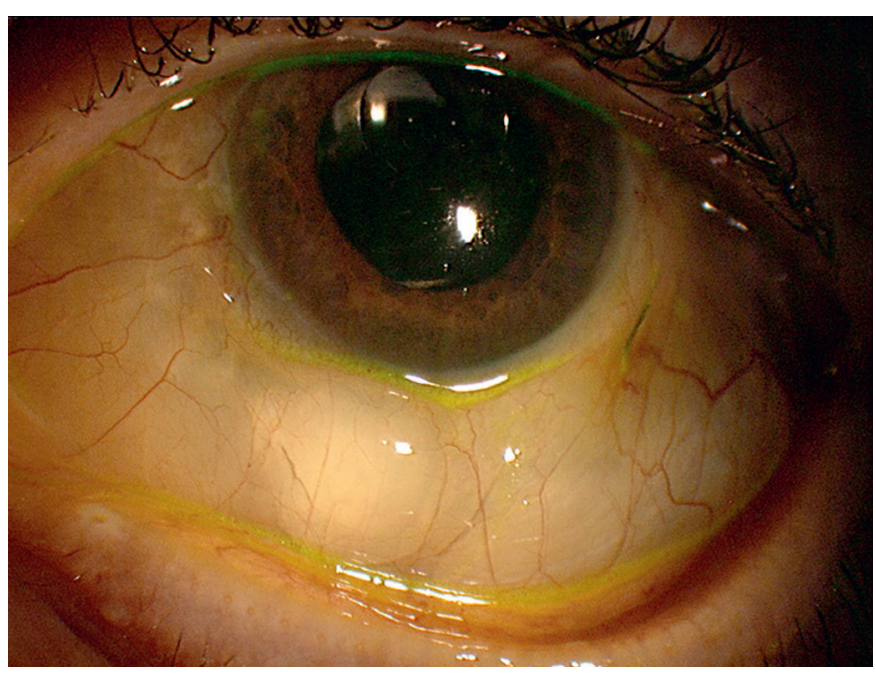

Abb. 1. Erscheinungsbild der Konjunktiva 3 Wochen nach Pars-plana-Vitrektomie und Silikonölinjektion. Im inferonasalen Quadranten ist eine große Silikonölblase im subkonjunktivalen Raum erkennbar.

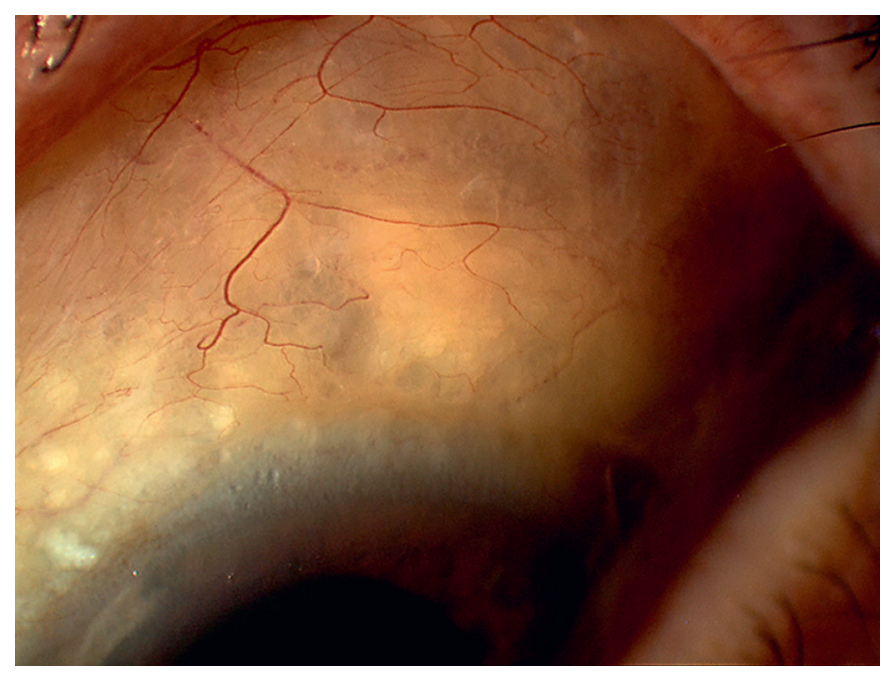

Abb. 2. Erscheinungsbild der Konjunktiva 2 Monate nach Pars-plana-Vitrektomie und Silikonölinjektion. Im superotemporalen Quadranten finden sich unzählige Silikonöl-Mikrobläschen, die den subkonjunktivalen Raum ausfüllen.

bereits gebildet und die Leckage ist möglicherweise auf das Vorliegen einer Pore in der Kapsel oder einen zu weiten Skleratunnel, durch den das Öl austreten konnte, zurückzuführen.

Ein Ersatz des Röhrchens in der Hinterkammer, um einen Kontakt mit dem Silikonöl zu verhindern, wäre vermutlich die bessere therapeutische Option gewesen. Da die Sehschärfe des Patienten «keine Lichtwahrnehmung» war, entschieden wir uns jedoch für eine konservative Behandlung, um eine stärkere Manipulation zu vermeiden.

Die nahtlose transkonjunktivale 23-Gauge-Vitrektomie mit Injektion von Silikonöl ist in der vitreoretinalen Chirurgie seit ihrer Einführung 2005 allgemein anerkannt [8]. Zwar kommt es nach einer Vitrektomie häufig zu einer begrenzten subkonjunktivalen 
Silikonöl-Leckage, doch wurde bislang keine massive SilikonölMigration berichtet. Einigen Berichten zufolge liegt die Inzidenz von kleinen subkonjunktivalen Silikonöl-Blasen bei 8 bis $10 \%$, und bisweilen kommt es zu leichten postoperativen Beschwerden [4]. In der Spaltlampenuntersuchung sind Silikonblasen normalerweise schwer erkennbar. Histopathologische Untersuchungen haben gezeigt, dass in 30\% der Augen nach nahtloser PPV kleine Silikontropfen zu finden sind [5]. Unsere Untersuchungen mittels Visante OCT nach PPV mit Silikonöl-Injektion bestätigen diese Angabe (Daten nicht veröffentlicht).

Möglicherweise beeinflusst die Viskosität des Silikonöls seine Leckage durch die Sklerotomie. Seit dem Aufkommen der PPV mittels Mikroinzision bevorzugen die Operateure meist niedrig visköses Silikonöl, das sich durch die dünne Kanüle leichter einbringen und entfernen lässt. Je niedriger die Viskosität, desto niedriger ist jedoch die Oberflächenspannung, sodass niedrig viskoses Silikonöl leichter eine nicht durch Nähte verschlossene Sklerotomie passieren kann als Silikonöl mit höherer Viskosität.

In den vorliegenden Berichten über eine subkonjunktivale Silikonöl-Migration wurde Silikonöl mit einer Viskosität von 1000 Zentistokes verwendet oder die Viskosität war nicht angegeben. Die Verwendung von Silikonöl mit höherer Viskosität kann vermutlich eine Leckage verhindern.

Ein weiterer wichtiger Aspekt, den es zu berücksichtigen gilt, ist der Durchmesser des Loches, durch das das Silikonöl hindurchtreten muss. Die Mikrokanüle hat einen Innendurchmesser von 0,65 mm und einen Außendurchmesser von 0,75 mm. Demgegenüber beträgt der Innendurchmesser des Röhrchens der AhmedDrainage 0,3 $\mathrm{mm}$ und der Außendurchmesser 0,63 mm. Im Vergleich zu den bei der PPV mit Mikroinzision verwendeten Kanülen haben GDD engere Durchmesser. Infolgedessen kann das Silikonöl leichter durch Sklerotomien migrieren als durch GDDRöhrchen, sofern beide offen sind.

Eine der Hauptsorgen im Hinblick auf eine Silikonöl-Leckage ist die Abdichtung der Wunde. Es wurden einige Empfehlungen ausgesprochen, um eine dichte Selbstabdichtung der Wunde am Ende der Operation zu erreichen: Inzision in einem Winkel von etwa $30^{\circ}$ durch die Augenwand, möglichst geringe Dehnung der Sklera während der chirurgischen Manöver, Erreichen eines normalen IOP-Werts vor Entfernung der Mikrokanüle oder Verwendung von bipolarer Kauterisation an der Konjunktiva über der Wunde [6]. Die sicherste Methode, um eine Leckage von Silikonöl zu verhindern, ist jedoch der Verschluss aller Sklerotomien durch Nähte. Manchmal wird eine Einzelknopfnaht belassen, die einige Tage später ambulant entfernt werden kann.

Maßnahmen zur Kontrolle der Silikonöl-Migration und -Leckage, wie beispielsweise eine Entfernung des Röhrchens oder eine Ligatur können mit einem hohen Risiko für einen Verlust der IOP-Kontrolle einhergehen. Eine weitere mögliche Option ist die Änderung der Röhrchenposition im inferotemporalen Quadranten der Vorderkammer. Da die Migration des Silikonöls oft erst nach Monaten auftritt, wird sie jedoch gemeinhin nicht durchgeführt, um die Manipulationen möglichst gering zu halten.

Versuche, das gesamte subkonjunktivale Silikonöl zu entfernen sind von begrenztem Erfolg, da unendlich viele Mikrotropfen des Öls das subkonjunktivale Gewebe infiltrieren und häufig fibröse Septen entstehen.

Insgesamt sprechen das Ausmaß der Silikonöl-Migration sowie der Zeitpunkt des Auftretens nach der Operation und die $360^{\circ}$ umfassende subkonjunktivale Lokalisation dafür, dass bei unserem Patienten eine nahtlose Sklerotomie oder ein großer Defekt der GGD-Kapsel die Ursache der Leckage war. In Fällen, in denen Silikonöl zum Einsatz kommt, empfiehlt es sich, die GDD-Implantate niederschwellig zu überprüfen und Sklerotomien mit Nahtverschluss zu verwenden.

\section{Danksagung}

Der vorliegende Fallbericht wurde vom Department of Ophthalmology, Sant Pau Hospital, unterstützt.

\section{Erklärung zu ethischen Konflikten}

Von dem Patienten wurde nach Aufklärung eine schriftliche Einverständniserklärung für die Veröffentlichung des vorliegenden Fallberichts und der dazugehörigen Bilder eingeholt. Die in dieser Arbeit verwendeten und/ oder analysierten Datensätze sind auf begründete Anfrage vom Korrespondenzautor erhältlich.

\section{Offenlegung}

Die Autoren erklären, dass keine Interessenkonflikte bestehen.

\section{Beiträge der einzelnen Autoren}

J. T. und J. I. V. nahmen die klinische Beurteilung, die Analyse und die Auswertung der Patientendaten im Hinblick auf die Augenmanifestationen vor. J. T. und J. I. V. waren die wichtigsten Beitragenden bei der Erstellung des Manuskripts. Alle Autoren haben die finale Fassung des Manuskripts gelesen und freigegeben.

\section{Literatur}

$\checkmark 1$ Hyung SM, Min JP: Subconjunctival silicone oil drainage through the Molteno implant. Korean J Ophthalmol 1998;12:73-75.

2 Chan KC, Tarasewicz DG, Lin SG: Subconjunctival migration of silicone oil through a Baerveldt pars plana glaucoma implant. Br J Ophthalmol 2005;89:240-241.

3 Nazemi PP, Chong LP, Varma R, et al.: Migration of intraocular silicone oil into the subconjunctival space and orbit through an Ahmed glaucoma valve. Am J Ophthalmol 2001;132: 929-931.

4 Siqueira RC, Gil AD, Jorge R: Retinal detachment surgery with silicone oil injection in transconjunctival sutureless 23-gauge vitrectomy. Arq Bras Oftalmol 2007;70:905-909.

5 Cunha LP, Primiano Júnior HP, Nakashima A, et al.: Subconjunctival deposit of silicone oil after vitreoretinal surgery. Arq Bras Oftalmol 2007;70:589-592.
6 Erakgun T, Egrilmez S: Surgical outcomes of transconjunctival sutureless 23-gauge vitrectomy with silicone oil injection. Indian J Ophthalmol 2009;57:105-109.

7 Gorovoy IR, Stewart JM: $360^{\circ}$ subconjunctival silicone oil after unsutured 23 -gauge vitrectomy. Eye 2013;27:894-895.

8 Eckardt C: Transconjunctival sutureless 23gauge vitrectomy. Retina 2005;25:208-211. 